\title{
Matrix-assisted laser desorption/ionization time-of-flight intact cell mass spectrometry to detect emerging pathogenic Candida species ${ }^{2 / 3}$
}

\author{
Cledir Santos ${ }^{\mathrm{a}}$, Nelson Lima ${ }^{\mathrm{a}}$, Paula Sampaio ${ }^{\mathrm{b}}$, Célia Pais ${ }^{\mathrm{b}, *}$ \\ ${ }^{a}$ IBB-Institute for Biotechnology and Bioengineering, Centre of Biological Engineering, University of Minho, Braga, Portugal \\ ${ }^{\mathrm{b}}$ Centre of Molecular and Environmental Biology (CBMA), University of Minho, Campus of Gualtar, 4710-057 Braga, Portugal
}

Received 18 May 2011; accepted 8 July 2011

\begin{abstract}
Matrix-assisted laser desorption/ionization time-of-flight intact cell mass spectrometry (MALDI-TOF-ICMS) was used to differentiate pathogenic Candida species, difficult to identify by traditional methods such as growth and biochemical reactions. Results showed that species complexes like C. parapsilosis, C. orthopsilosis, and C. metapsilsosis, and very closely related species like $C$. glabrata and C. bracarensis, and C. albicans and C. dubliniensis could be clearly separated. MALDI-TOF-ICMS stands out as a promising tool for the rapid detection of emerging pathogens.
\end{abstract}

(C) 2011 Elsevier Inc. All rights reserved.

Keywords: Candida; C. parapsilosis complex; MALDI-TOF ICMS

The clinical impact of fungal infections has greatly increased in the last years, particularly in immunocompromised hosts. Yeasts belonging to the genus Candida have emerged as the major opportunistic pathogens in these patients and currently constitute the fourth most common cause of nosocomial infections in intensive care units (Perlroth et al., 2007; Rueping et al., 2009). Candida albicans is the species most commonly isolated, although others, such as C. tropicalis, C. krusei, C. parapsilosis, and $C$. glabrata, have increasingly been recognized as pathogens with a wide distribution (Pfaller and Diekema 2007, Perlroth et al., 2007). Additionally, new species have been described, for example, $C$. dubliniensis related to C. albicans (Sullivan et al., 1995); C. orthopsilosis and C. metapsilosis, very closely related to C. parapsilosis (Tavanti et al., 2005); and C. bracarensis, phenotypically similar to C. glabrata (Correia et al., 2006), which are emerging as potential pathogens.

\footnotetext{
Research leading to these results received funding from the European Community's Seventh Framework Program (FP7 2007-2013), Research Infrastructures Action, under grant agreement no. FP7-228310 (EMbaRC project).

* Corresponding author. Tel.: +351-253604312; fax: +351-253678987.

E-mail address: cpais@bio.uminho.pt (C. Pais).
}

Early identification of the species causing infection is crucial for adequate treatment since the antifungal susceptibility profile differs greatly among species. Moreover, the time needed for the identification of the pathogen is an important determinant of infection-related mortality rates in hospitalised patients. The methods currently used for yeast identification in clinical laboratories are not able to distinguish emerging pathogenic species and, additionally, are either time consuming and/ or require considerable expertise.

Several reports have shown that matrix-assisted laser desorption/ionisation time-of-flight intact cell mass spectrometry (MALDI-TOF ICMS) is suitable for the fast and reliable identification of pathogenic microorganisms (Barbuddhe et al., 2008; Claydon et al., 1996; Degand et al 2008; Mellman et al., 2008), including filamentous fungi (Santos et al., 2010) and yeasts (Marklein et al., 2009; Qian et al., 2008; Putignani et al., 2011; Stevenson et al., 2010; van Veen et al., 2010). The performance and cost analysis of MALDI-TOF ICMS for routine identification of yeast were recently evaluated by Dhiman et al. (2011) who concluded that this is an accurate, rapid, and cost-effective technique that could be applied in a medical microbiology laboratory for identification of yeast clinical isolates. Therefore, the aim of the present work was to use MALDI-TO-ICMS for the identification of Candida sp. 
clinical isolates and to evaluate its ability for differentiating newly described species impossible to detect by the conventional phenotypic methods used in clinical laboratories.

Sixty-seven clinical isolates, belonging to 9 Candida species, namely, C. albicans, C. tropicalis, C. kruzei, C. parapsilosis, C. orthopsilosis, C. matapsilosis, C. lusitaniae, C. glabrata, and C. bracarensis, were analysed. Two strains of Lodderomyces elongisporus and 4 Saccharomyces cerevisiae isolates were also included in the study. Candida clinical isolates were obtained from several hospitals and health institutions and collected from blood, bronchial secretions, urine, and vaginal exudates. All isolates were identified by identification cards (BioMerieux, France) at the institutions of origin, and most were confirmed by 26S rDNA and ITS sequencing at the Department of Biology, University of Minho. The isolates were cryopreserved at $-80{ }^{\circ} \mathrm{C}$. Yeast cells were grown on YEPD agar medium plates for $24 \mathrm{~h}$, at $37{ }^{\circ} \mathrm{C}$. A loopful of yeast cells was directly transferred from the culture medium onto each position of the 48-well flex target plate, and $0.5 \mu \mathrm{L}$ of $25 \%$ formic acid was immediately mixed with the yeast. After evaporation, $0.5 \mu \mathrm{L}$ matrix solution $(75 \mathrm{mg} / \mathrm{mL} 2,5-$ dihydroxybenzoic acid in ethanol/water/acetonitrile [1:1:1] with $0.03 \%$ trifluoroacetic acid) was added and gently mixed. All sample mixtures were air dried at room temperature. Each isolate was spotted in triplicate.

Analyses were performed on an Axima LNR system (Kratos Analytical, Shimadzu, Manchester, UK) equipped with a nitrogen laser $(337 \mathrm{~nm})$. The mass range from 2,000 to $20,000 \mathrm{Da}$ was recorded by using the linear mode. Escherichia coli ribosomal proteins were used for external calibration of the spectra. Spectra were exported to the SARAMISTM software package (Spectral Archiving and Microbial Identification System, AnagnosTec, PostdamGolm, Germany, http://www.anagnostec.eu) where the final identifications were achieved. Cluster analysis of the MALDI-TOF ICMS mass spectral data was performed using the SARAMISTM database by comparing database peak lists of individual samples with SuperSpectra and/or reference spectra.
The mass spectra obtained presented peaks ranging from 2,000 to $12,000 \mathrm{Da}$, with the most intense within the range of 5,000 to 7,000 Da. Mass spectra of the different Candida species analyzed showed distinctive features that could be used for species differentiation, and examples of distinctive spectra are shown in Fig. 1.

Initially, the isolates of $C$. metapsilosis, C. orthopsilosis, and $C$. bracarensis could not be identified since spectra for appropriate reference strains were not available in the database. As the identity of the isolates analysed in this study had been previously confirmed by D1/D2 26S rDNA and ITS sequencing, a reference spectrum for each was introduced into the database and subsequent comparison with the unidentified species resulted in a matching profile for all the isolates.

The species presenting the highest number of peaks were the C. glabrata and C. bracarensis isolates, which both showed an additional intense peak at around 11,000 Da. C. parapsilosis and C. metapsilosis isolates were the ones yielding a lower number of peaks. Additionally, the closely related species, C. parapsilosis, C. metapsilosis, and C. orthopsilosis, and C. glabrata and C. bracarensis, presented similar but yet distinguishable profiles (Fig. 1).

L. elongisporus, a species hypothesized to be $C$. parapsilosis teleomorph (James et al., 1994) and considered very closely related by sequence analysis (Butler et al., 2009), presented a completely distinct profile (Fig. 1). This observation is particularly interesting since L elongisporus has been recently described in cases of bloodstream infection misidentified as C. parapsilosis (Lockhart et al., 2008a).

C. glabrata and C. bracarensis presented similar spectra. The $S$. cerevisiae profile was distinctive, although peaks in the same spectral region as for C. glabrata and C. bracarensis (between 4,000 and 6,600 Da) could be observed. These data confirm the close genomic and proteomic relation between $S$. cerevisiae and C. glabrata, as described in the literature (Roetzer et al., 2011), but still the 3 species appear as distinct.

Cluster analysis of the MALDI-TOF ICMS mass spectral data included all peaks obtained from 2,000 to 20,000 Da.

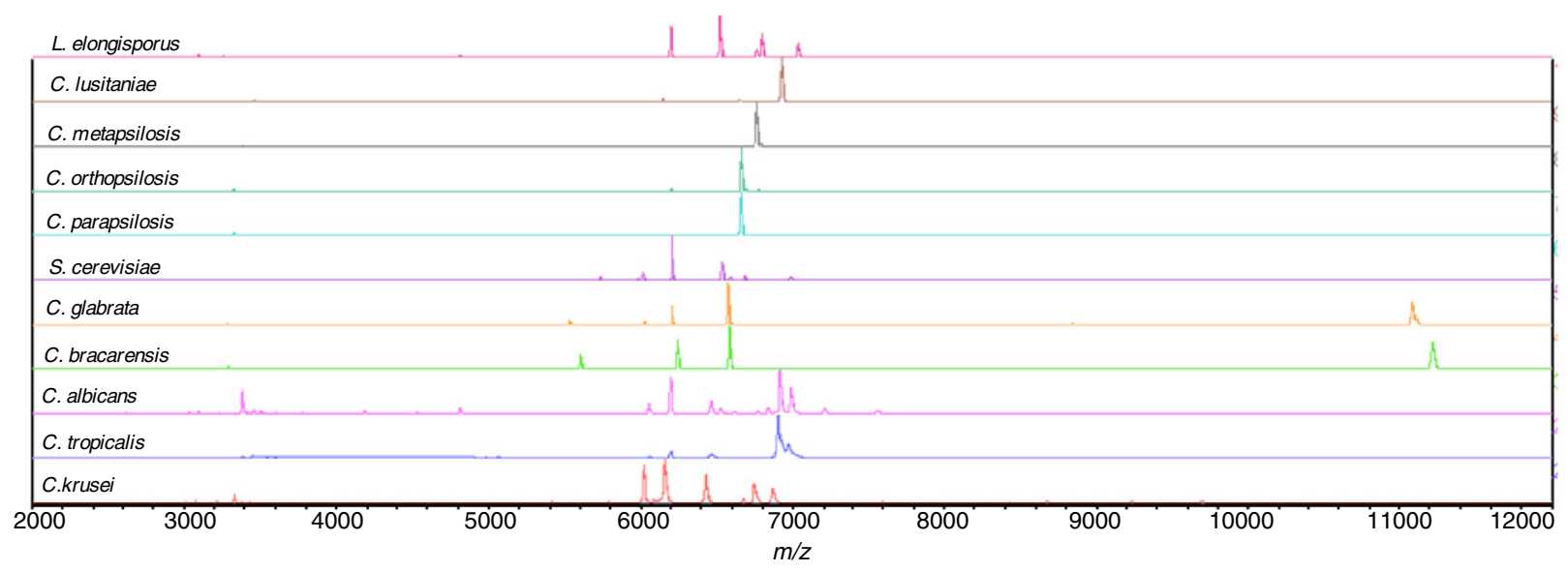

Fig. 1. Examples of representative MALDI-TOF ICMS spectra of the yeast species analysed in this study. 


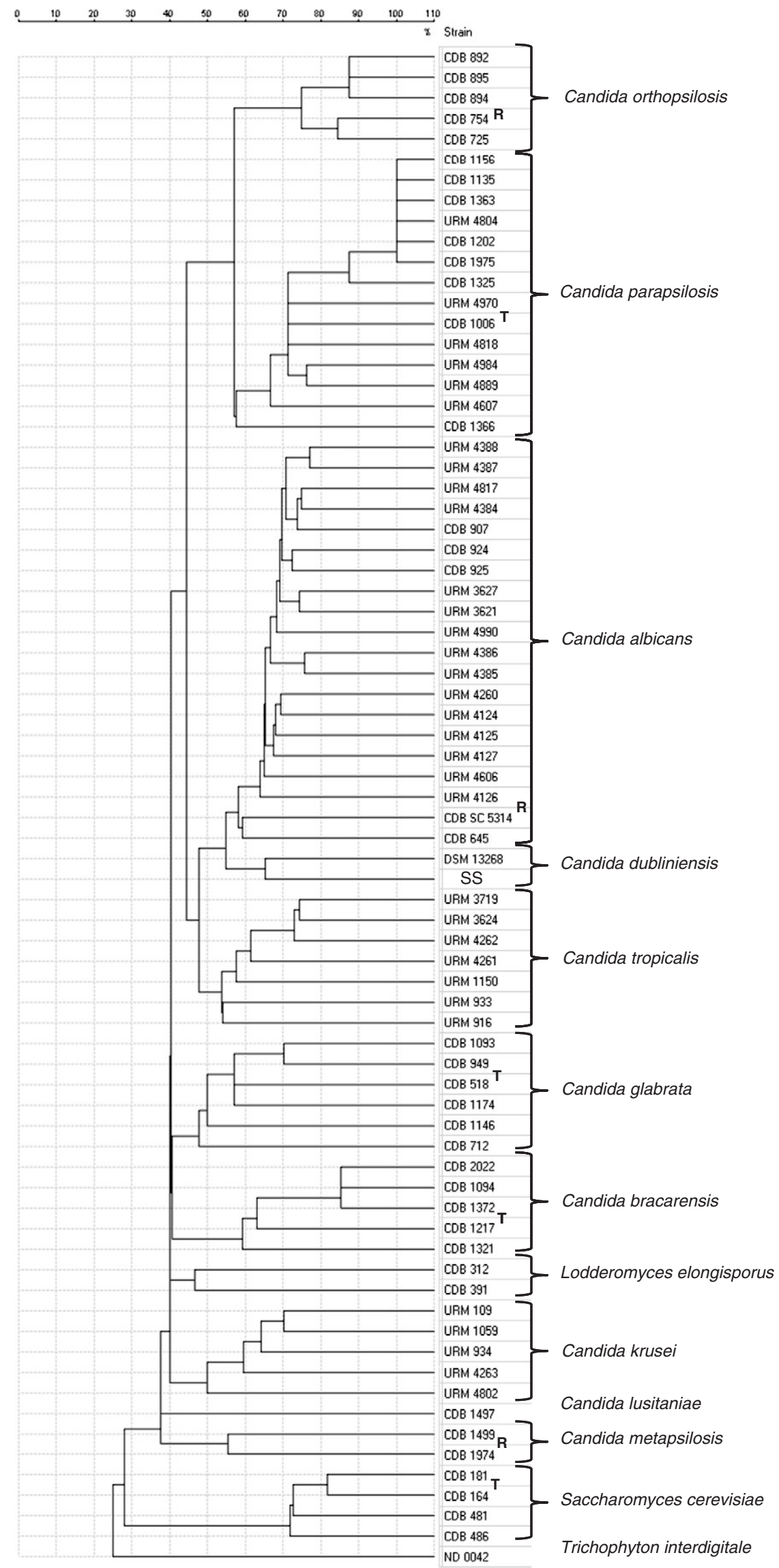


Mass spectral signatures allowed the grouping of all isolates into clusters according to their species designation (Fig. 2). These results are in accordance with the ones reported previously (Bader et al., 2010; Marklein et al., 2009; Qian et al., 2008; Stevenson et al., 2010). At a threshold of about $48 \%$ similarity, all different species were clearly distinguished except the pairs $C$. parapsilosis/C. orthopsilosis and $C$. albicans/C. dubliniensis, which could only be distinguished at a threshold of $57 \%$ and $52 \%$, respectively. C. dubliniensis isolates were not analysed in the present work but spectra, obtained from the SARAMIS database, were included in the analysis. Spectra obtained for all isolates belonging to the same species grouped together, presenting a very close profile.

All of the closely related species, C. albicans and C. dubliniensis; C. parapsilosis, C. metapsilosis, and C. orthopsilosis; as well as C. glabrata and C. bracarensis, could be clearly distinguished. Also, $S$. cerevisiae and L. elongisporus species were distinctly separated. Due to their close phylogenetic relationship with the species most commonly found, the new species mentioned above are often misidentified and MALDI-TOF ICMS analysis can be a valuable tool for their early and unequivocal detection. This is particularly important since in recent $C$. parapsilosis surveys a growing importance of $C$. orthopsilosis has been reported (Gomez-Lopez et al., 2008; Lockhart et al., 2008b).

MALDI-TOF ICMS appears to be a powerful highthroughput proteomic technique to discriminate closely related Candida species, which is feasible otherwise only by molecular biology techniques. The equipment is straightforward to operate and inexpensive on an individual sample basis. The rapid identification of uncommon yeast species that are emerging as human pathogens may be accurately achieved provided that a complete and quality-controlled database is available.

\section{Acknowledgments}

Thanks are due to Reginaldo Lima-Neto and Rejane P. Neves from the URM Culture Collection of the Federal University of Pernambuco (Brazil) for supplying part of the strains used in this study.

\section{References}

Bader O, Weig M, Taverne-Ghadwal L, Lugert R, Groß U, Kuhns M (2010) Improved clinical laboratory identification of human pathogenic yeasts by matrix-assisted laser desorption ionization time-of-flight mass spectrometry. Clin Microbiol Infect, doi:10.1111/j.1469-0691.2010.03398.

Barbuddhe SB, Maier T, Schwarz G, Kostrzewa M, Hof H, Domann E, Chakraborty T, Hain T (2008) Rapid identification and typing of Listeria species by matrix-assisted laser desorption ionizationtime of flight mass spectrometry. Appl Environ Microbiol 74: $5402-5407$.
Butler G, Rasmussen MD, Lin MF, Santos MA, Sakthikumar S, Munro CA, Rheinbay E, Grabherr M, Forche A, Reedy JL, Agrafioti I, Arnaud MB, Bates S, Brown AJ, Brunke S, Costanzo MC, Fitzpatrick DA, de Groot PW, Harris D, Hoyer LL, Hube B, Klis FM, Kodira C, Lennard N, Logue ME, Martin R, Neiman AM, Nikolaou E, Quail MA, Quinn J, Santos MC, Schmitzberger FF, Sherlock G, Shah P, Silverstein KA, Skrzypek MS, Soll D, Staggs R, Stansfield I, Stumpf MP, Sudbery PE, Srikantha T, Zeng Q, Berman J, Berriman M, Heitman J, Gow NA, Lorenz MC, Birren BW, Kellis M, Cuomo CA (2009) Evolution of pathogenicity and sexual reproduction in eight Candida genomes. $\mathrm{Na}$ ture 459:657-662.

Claydon MA, Davey SN, Edwards J, Gordon DB (1996) The rapid identification of intact microorganisms using mass spectrometry. Nat Biotechnol 14:1584-1586.

Correia A, Sampaio P, James S, Pais C (2006) Candida bracarensis sp. nov., a novel anamorphic yeast species phenotypically similar to Candida glabrata. Int J Syst Evol Microbiol 56:313-317.

Degand N, Carbonnelle E, Dauphin B, Beretti JL, Le Bourgeois M, Segonds C, Berche P, Nassif X, Ferroni A (2008) Matrix-assisted laser desorption ionization-time of flight mass spectrometry for identification of nonfermenting Gram-negative bacilli isolated from cystic fibrosis patients. J Clin Micobiol 46:3361-3367.

Dhiman N, Hall L, Wohlfiel SL, Buckwalter SP Wengenack NL (2011) Performance and cost analysis of matrix-assisted laser desorption ionization-time of flight mass spectrometry for routine identification of yeast. J Clin Micobiol 49:1614-1616.

Gomez-Lopez A, Alastruey-Izquierdo A, Rodriguez D, Almirante B, Pahissa A, Rodriguez-Tudela JL, Cuenca-Estrella M, Barcelona Candidemia Project Study Group (2008) Prevalence and susceptibility profile of Candida orthopsilosis and Candida metapsilosis. Results from population based surveillance of candidemia in Spain. Antimicrob Agents Chemother 52:1506-1509.

James SA, Collins MD, Roberts IN (1994) The genetic relationship of Lodderomyces elongisporus to other ascomycete yeast species as revealed by small subunit rRNA gene sequences. Lett Appl Microbiol 19:308-311.

Lockhart S, Messer S, Pfaller M, Diekema DJ (2008a) Lodderomyces elongisporus masquerading as Candida parapsilosis as a cause of bloodstream infections. J Clin Microbiol 46:374-376.

Lockhart S, Messer S, Pfaller M, Diekema DJ (2008b) Geographic distribution and antifungal susceptibility of the newly described species Candida orthopsilosis and Candida metapsilosis in comparison to the closely related species Candida parapsilosis. J Clin Microbiol 46:2659-2664.

Marklein G, Josten M, Klanke U, Müller E, Horré R, Maier T, Wenzel T, Kostrzewa M, Bierbaum G, Hoerauf A, Sahl HG (2009) Matrix-assisted laser desorption ionization-time of flight mass spectrometry for fast and reliable identification of clinical yeast isolates. J Clin Microbiol 47:2912-2917.

Mellman A, Cloud J, Maier T, Keckevoet U, Ramminger I, Iwen P, Dunn J, Hall G, Wilson D, LaSala P, Kostrzewa M, Harmsen D (2008) Evaluation of matrix-assisted laser desorption ionization-time of flight mass spectrometry in comparison to $16 \mathrm{~S}$ rRNA gene sequencing for species identification of non fermenting bacteria. J Clin Microbiol 46: 1946-1954

Perlroth J, Choi B, Spellberg B (2007) Nosocomial fungal infections: epidemiology, diagnosis, and treatment. Med Mycol 45:321-346.

Pfaller M, Diekema DJ (2007) Epidemiology of invasive candidiasis: a persistent public health problem. Clin Microbiol Rev 20:133-163.

Putignani L, Del Chierico F, Onori M, Mancinelli L, Argentieri M, Bernaschi P, Coltella L, Lucignano B, Pansani L, Ranno S, Russo C, Urbani A, Federicibd G, Menichella D (2011) MALDI-TOF mass

Fig. 2. MALDI-TOF ICMS spectra-based dendrogram of the yeast isolates studied in this work. The spectrum of the dermatophyte fungus Trichophyton interdigitale was used as outgroup. $\mathrm{CDB}=$ Collection Department of Biology; $\mathrm{T}=$ Type strain; $\mathrm{R}=$ reference strain; $\mathrm{SS}=$ Super septrum. 
spectrometry proteomic phenotyping of clinically relevant fungi. Mol Biosyst, doi:10.1039/c0mb00138d.

Qian J, Cutler JE, Cole RB, Cai Y (2008) MALDI-TOF mass signatures for differentiation of yeast species, strain grouping and monitoring of morphogenesis markers. Anal Bioanal Chem 392:439-449.

Roetzer A, Gabaldón T, Schüller C (2011) From Saccharomyces cerevisiae to Candida glabrata in a few easy steps: important adaptations for an opportunistic pathogen. FEMS Microbiol Lett 314:1-9.

Rueping MJ, Vehreschild JJ, Cornely OA (2009) Invasive candidiasis and candidemia: from current opinions to future perspectives. Expert Opin Invest Drugs 18:735-748.

Santos C, Paterson RMR, Venâncio A, Lima N (2010) Filamentous fungal characterisations by matrix-assisted laser desorption/ionisation time of flight mass spectrometry. J Appl Microbiol 108: $375-385$.
Stevenson LG, Drake SK, Shea YR, Zelazny AM, Murray PR (2010) Evaluation of matrix-assisted laser desorption ionization-time of flight mass spectrometry for identification of clinically important yeast species. J Clin Micobiol 48:3482-3486.

Sullivan DJ, Westerneng TJ, Haynes KA, Bennett DE, Coleman DC (1995) Candida dubliniensis sp. nov.: phenotypic and molecular characterization of a novel species associated with oral candidosis in HIV-infected individuals. Microbiology 141:1507-1521.

Tavanti A, Davidson AD, Gow NAR, Maiden MCJ, Odds FC (2005) Candida orthopsilosis and Candida metapsilosis spp. nov. to replace Candida parapsilosis groups II and III. J Clin Microbiol 43:284-292.

van Veen SQ, Claas EC, Kuijper EJ (2010) High-throughput identification of bacteria and yeast by matrix-assisted laser desorption ionization-time of flight mass spectrometry in conventional medical microbiology laboratories. J Clin Microbiol 48:900-907. 